\title{
Characteristic Exponents for a Viscous Fluid Subjected to Time Dependent Forces
}

David Ruelle

I.H.E.S., F-91440 Bures-sur-Yvette, France

\begin{abstract}
We consider a viscous incompressible fluid enclosed in a bounded region of $\mathbb{R}^{2}$ or $\mathbb{R}^{3}$, and subjected to time dependent forces. Using bound state estimates for the Schrödinger operator, we obtain rigorous bounds for the characteristic exponents, entropy (Kolmogorov-Sinai invariant), and Hausdorff dimension of attracting sets. Our methods are of potential use for more general time evolutions described by nonlinear partial differential equations.
\end{abstract}

\section{Introduction}

In an earlier paper (Ruelle [32]) some rigorous inequalities on the characteristic exponents for the Navier-Stokes time evolution have been obtained. These inequalities were based on estimates for the eigenvalues of Schrödinger operators, and have been subsequently improved by Lieb [18]. Using other methods, Constantin and Foias [4] have also investigated the characteristic exponents for Navier-Stokes (in the 2-dimensional case).

From the estimates on characteristic exponents one obtains rigorous bounds on the Kolmogorov-Sinai invariant (rate of creation of information) and the Hausdorff dimension of attracting sets. Such estimates were given in [32] and [4]. We indicate below the best bounds currently known, based on the results of Lieb [18] (see Eqs. (14)-(17)). This improves in particular the bounds on the Hausdorff dimension of attracting sets obtained by Constantin and Foias [4]. Explicit results are also obtained for a 2-dimensional convection problem.

One novelty of the present paper is to lift the requirement that the forces acting on the fluid be time independent.

\section{Definition of the Characteristic Exponents}

We consider a time evolution equation

$$
\frac{d}{d t} x=F(x, t)
$$


in a separable Hilbert space $M^{1}$ and assume that the general solution $x=f\left(x_{0}, t\right)$ is well defined for initial condition $x_{0}$ in a suitable open subset $U \subset M$ and $t \geqq 0$. We also assume the existence of the linear operator $T\left(x_{0}, t\right): M \mapsto M$ obtained by taking the derivative of $f\left(x_{0}, t\right)$ with respect to the first argument, i.e., $T\left(x_{0}, \cdot\right)=D_{x_{0}} f$. The growth of a small perturbation $\xi$ of the initial condition is described by $\xi(t)=T\left(x_{0}, t\right) \xi_{0}$. Alternatively, $\xi$ satisfies the linearized equation

$$
\frac{d}{d t} \xi=\left(D_{x(t)} F\right) \xi
$$

Note that the linear Eq. (2) is time dependent even if $F$ does not explicitly depend on $t$. We define the characteristic exponent $\lambda\left(x_{0}, \xi_{0}\right)$ as the following limit if it exists:

$$
\lambda\left(x_{0}, \xi_{0}\right)=\lim _{t \rightarrow \infty} \frac{1}{t} \log \|\xi(t)\| .
$$

We shall first discuss the case where the right-hand side of $(1)$ does not depend explicitly on $t: F(x, t)=F(x)$. We write then $f(x, t)=f^{t} x$ and $T(x, t)=T_{x}^{t}$ and we have, where defined,

$$
\begin{gathered}
f^{s+t}=f^{s} \circ f^{t}, f^{0}=\text { identity }, \\
T_{x}^{s+t}=T_{f^{t} x}^{s} T_{x}^{t}, T_{x}^{0}=\mathbb{1} .
\end{gathered}
$$

If $M$ is finite dimensional ${ }^{2}$ and $\varrho$ is a probability measure on $M$, invariant under time evolution (i.e., under the $f^{t}$, all $t \geqq 0$ ), the multiplicative ergodic theorem of Oseledec ${ }^{3}$ implies the existence of the limit (3) for $\varrho$-almost all $x_{0}$ (and all $\xi_{0}$ ). Furthermore $^{4}$

$$
\lim _{t \rightarrow \infty}\left(\left(T_{x}^{t}\right)^{*} T_{x}^{t}\right)^{1 / 2 t}=\Lambda_{x}
$$

also exists almost everywhere and the characteristic exponents $\lambda(x, \cdot)$ are identical with the logarithms $\lambda_{i}(x)$ of the eigenvalues of $\Lambda_{x}$. If $\varrho$ is ergodic, the characteristic exponents are almost everywhere constant.

The multiplicative ergodic theorem does not extend without further conditions to the case where $M$ has infinite dimension. In fact one can find a bounded operator $T$ such that $\left(\left(T^{n}\right)^{*} T^{n}\right)^{1 / 2 n}$ does not tend to a limit when $n \rightarrow \infty{ }^{5}$. However, the multiplicative ergodic theorem holds if the operators $T_{x}^{t}$ are compact ${ }^{6}$ (i.e., completely continuous: they can be approached in norm by finite rank operators). This is sufficient for applications to the Navier-Stokes time evolution.

\footnotetext{
1 In the Appendix we allow $M$ to be a Hilbert manifold. Here we take $M$ linear for notational simplicity

2 We also assume that $f, T$ depend continuously on $x$ and $t$. Much weaker conditions are actually needed

3 See Oseledec [26], Raghunathan [27], Ruelle [30]

4 In (4) we denote by $T^{*}$ the adjoint of $T$; since $T^{*} T$ is positive, its positive $\operatorname{root}\left(T^{*} T\right)^{1 / 2 t}$ is well defined

5 This was shown to me by T. Körner (Cambridge). This example contradicts the multiplicative ergodic theorem when the support of $\varrho$ is reduced to one point (and the time is discrete rather than continuous)

6 See Ruelle [31], Mañé [24]; Mañé's results extend to Banach spaces
} 


\section{The Time Dependent Case}

To treat the case where the right-hand side of (1) depends explicitly on $t$ we have to make some statistical assumption on its time dependence. Here we discuss the situation formally, the appropriate theorems are quoted in the Appendix, and their applicability to physics is discussed in Sect. 6 .

Let $(S, \sigma)$ be a probability space and $\left(\mathbf{g}^{t}\right)_{t \geq 0}$ a semi-group of measure-preserving transformations of $S$ with respect to which $\sigma$ is ergodic. We shall assume that $F(x, t)=\mathbf{F}\left(x, \mathbf{g}^{t} y_{0}\right)$ for some $y_{0} \in S$ and some function $\mathbf{F}: M \times S \rightarrow M$.

For instance we may take for $S$ the $k$-torus $\left\{\left(y_{1}, \ldots, y_{k}\right) \bmod 2 \pi\right\}$, for $\sigma$ the Haar measure, and let $\mathbf{g}^{t}\left(y_{1}, \ldots, y_{k}\right)=\left(y_{1}+\omega_{1} t, \ldots, y_{k}+\omega_{k} t\right)$, (ergodicity is ensured if there is no relation $\sum n_{i} \omega_{i}=0$ with integers $n_{i}$ not all zero). This corresponds to quasiperiodic forces (periodic forces if $k=1$ ).

If the equation

$$
\frac{d}{d t} x=\mathbf{F}\left(x, \mathbf{g}^{t} y_{0}\right)
$$

has a well defined general solution $x=\mathbf{f}\left(x_{0}, y_{0}, t\right)$, we write $\mathbf{f}^{\mathbf{t}}\left(x_{0}, y_{0}\right)=\left(\mathbf{f}\left(x_{0}, y_{0}, t\right)\right.$, $\left.\mathbf{g}^{t} y_{0}\right)$, and we have $\mathbf{f}^{s+t}=\mathbf{f}^{\mathbf{s}} \circ \mathbf{f}^{t}, \mathbf{f}^{0}=$ identity. We assume that $\left(\mathbf{f}^{t}\right)$ leaves invariant a probability measure $\varrho$ on $M \times S$ and that the projection $M \times S \rightarrow S$ maps $\varrho$ to $\sigma$. Let also $\mathbf{T}_{(x, y)}^{t}$ be the derivative of $\mathbf{f}(x, y, t)$ with respect to the first argument, then

$$
\mathbf{T}_{(x, y)}^{s+t}=\mathbf{T}_{\mathbf{f}^{t}(x, y)}^{s} \mathbf{T}_{(x, y)}^{t}, \mathbf{T}_{(x, y)}^{0}=\mathbb{1} .
$$

The multiplicative ergodic theorem may now be applied to define characteristic exponents ${ }^{7}$. In particular

$$
\lim _{t \rightarrow \infty}\left(\left(\mathbf{T}_{(x, y)}^{t}\right) * \mathbf{T}_{(x, y)}^{t}\right)^{1 / 2 t}=\Lambda_{(x, y)}
$$

exists for $\varrho$-almost all $x, y$.

To proceed we disintegrate the measure $\varrho$ by writing $\int \varrho(d x d y)=\int \varrho_{y}(d x) \sigma(d y)$, where the probability measure $\varrho_{y}$ on $M$ is defined for $\sigma$-almost all $y$. We see now that for $\sigma$-almost all $y$, we can revert to the study of (1) (with $F(\cdot, t)=\mathbf{F}\left(\cdot, \mathbf{g}^{t} y\right)$ ), and obtain the existence of the limit (3) and of

$$
\Lambda_{x}=\lim _{t \rightarrow \infty}\left(T(x, t)^{*} T(x, t)\right)^{1 / 2 t}
$$

for $\varrho_{y}$-almost all $x$. If $\varrho$ is $\mathbf{f}$-ergodic (this can be achieved by decomposition) then the characteristic exponents are almost everywhere constant.

In the quasiperiodic example the above condition "for $\sigma$-almost all $y$ " may be dropped (because in that case a $\left(\mathbf{g}^{t}\right)$ invariant measurable subset of $S$ is $\emptyset$ or $S$ ).

\section{Hausdorff Dimension and Entropy}

Assume again that the right-hand side $F$ of (1) is time independent, and let there be a compact invariant subset $K$ of $M$. The compactness of the derivative $T_{x}^{t}$ implies

7 See the Appendix for a precise statement 
that $K$ has finite Hausdorff dimension (Mallet-Paret $\left.[21]^{8}\right)$ ). An estimate of the Hausdorff dimension of $K$ in terms of characteristic exponents follows from the work of Frederikson, Kaplan and Yorke [9], Douady and Oesterlé [6], Mañé [23], and Ledrappier [16]. More precisely, let $\lambda_{1} \geqq \lambda_{2} \geqq \ldots$ be the decreasing sequence of characteristic exponents associated with the ergodic measure $\varrho$, define $c_{\varrho}(n)=\sum_{i}^{n} \lambda_{i}$ for integer $n \geqq 0$, and extend by linearity to the intervals $[n, n+1]$. Define the "Liapunov" dimension of $\varrho$ by

$$
\operatorname{dim}_{\Lambda}(\varrho)=\max \left\{s: c_{\varrho}(s) \geqq 0\right\} .
$$

Then (Ledrappier [16]) the Hausdorff dimension of $K$ satisfies:

$$
\left.\operatorname{dim}_{H} K \leqq \sup \operatorname{dim}_{\Lambda}(\varrho): \varrho \text { is ergodic and supp } \varrho \subset K\right\} \text {. }
$$

Ledrappier [16] also shows that $\operatorname{dim}_{\Lambda}(\varrho)$ is an upper bound to the Hausdorff dimension $\operatorname{dim}_{H}(\varrho)$, i.e., to the minimum of the Hausdorff dimensions of the sets $E$ such that $\varrho(E)=1$. This is an interesting quantity (L.-S. Young [37], Grassberger and Procaccia [12]) which can be measured experimentally (see Malraison, Atten, Bergé, and Dubois [22]) ${ }^{9}$.

Consider now the case of time dependent forces described by $F(x, t)=\mathbf{F}\left(x, \mathbf{g}^{t} y_{0}\right)$, where $\left(\mathbf{g}^{t} y_{0}\right)$ is distributed according to the measure $\sigma$. The characteristic exponents $\lambda_{1} \geqq \lambda_{2} \geqq \ldots$ associated with an ergodic measure $\varrho$ have been defined above ( $\varrho$ is a measure on $M \times S$, but the characteristic exponents correspond to expansion or contraction in $M$ only). We define $c_{\varrho}$ and $\operatorname{dim}_{\Lambda}(\varrho)$ as before. Suppose now that there is a compact set $K \subset M$ such that $\mathbf{f}^{t} K \times S \subset K \times S$ for $t \geqq 0$. There is then a compact set $K\left(y_{0}\right)$ such that ${ }^{10}$

$$
K\left(y_{0}\right) \times\left\{y_{0}\right\}=\bigcap_{t \geqq 0} \mathbf{f}^{t}\left(K \times \mathbf{g}^{-t} y_{0}\right)
$$

and its Hausdorff dimension satisfies (see Appendix):

$$
\begin{gathered}
\operatorname{dim}_{H} K\left(y_{0}\right) \leqq \sup \left\{\operatorname{dim}_{\Lambda}(\varrho): \varrho\right. \text { is ergodic, } \\
\operatorname{supp} \varrho \subset K \times S \text { and the projection on } S \text { maps } \varrho \text { to } \sigma\} .
\end{gathered}
$$

A dynamical system with given invariant probability measure $\varrho$ may always be considered as a source of information, and the rate of information creation is the Kolmogorof-Sinai invariant or entropy $h(\varrho)$. In particular we expect $h(\varrho)>0$ for a turbulent fluid (the system acts as a random number generator) even though its time evolution is deterministic. This is because of sensitive dependence on initial condition: two initial conditions which differ by an amount too small to be measured will differ by a measurable amount after some time $t$, so that new information on the system has become available.

In the case of time dependent forces, as discussed above, the rate of information creation by the system is the relative entropy $h_{\mathbf{f}}(\varrho)-h_{\mathbf{g}}(\sigma)=h(\varrho \mid \sigma)$. It is a general

8 This argument has been applied to the Navier-Stokes equation by Foias and Temam [8]

9 We note in passing that $\operatorname{dim}_{H} K$ is not in general equal to the supremum of the $\operatorname{dim}_{H} \varrho$ with supp $\varrho \subset K$ and $\varrho$ ergodic (see McCluskey and Manning [25])

10 We assume that $\mathrm{g}^{-t} y_{0}$ is well defined; this condition is weakened in the Appendix 
fact that this quantity is bounded above by the sum of the positive characteristic exponents (see Appendix):

$$
h_{\mathbf{f}}(\varrho)-h_{\mathbf{g}}(\sigma) \leqq \sum_{i: \lambda_{1}>0} \lambda^{i}(\varrho)
$$

In particular, for periodic or quasiperiodic forces we have $h(\sigma)=0$.

\section{Rigorous Inequalities}

Suppose that the time evolutions defined by (1), (2) make good sense (this has to be checked in individual cases). Suppose in particular that

$$
\mathscr{H}(x)=\frac{1}{2}\left(D_{x} F\right)+\frac{1}{2}\left(D_{x} F\right)^{*}
$$

is a well defined self-adjoint operator. Usually $\mathscr{H}(x)$ will be unbounded, but we assume that this operator is bounded above and that its spectrum is discrete, consisting of the eigenvalues $a_{1}(x) \geqq a_{2}(x) \geqq \ldots$, repeated according to (finite) multiplicity. Then it is shown in [32] that

$$
\sum_{1}^{n} \lambda_{i}(x(0)) \leqq \liminf _{t \rightarrow \infty} \frac{1}{t} \int_{0}^{t} \sum_{1}^{n} a_{i}(x(t)) .
$$

(This inequality is related to Lemma 2.1 of [16].) Notice that the estimate (7) forgets time correlations: the growth rate of a perturbation $\xi$ is estimated in terms of the maximum growth rate at each time. If $\varrho$ is an ergodic probability measure, (7) yields

$$
c_{\varrho}(n)=\sum_{1}^{n} \lambda_{i}(\varrho) \leqq \int \varrho(d x) \sum_{1}^{n} a_{i}(x)=\left\langle\sum_{1}^{n} a_{i}\right\rangle_{\varrho},
$$

and

$$
\sum_{i: \lambda_{i} \geqq 0} \lambda_{i}(\varrho) \leqq\left\langle\sum_{i: a_{i} \geqq 0} a_{i}\right\rangle_{\varrho}
$$

More generally (see [32])

$$
\sum_{i: \lambda_{i} \geqq 0}\left(\lambda_{i}(\varrho)\right)^{\gamma} \leqq\left\langle\sum_{i: a_{i} \geqq 0}\left(a_{i}\right)^{\gamma}\right\rangle_{\varrho}
$$

if $\gamma \geqq 1$. In fact, as noted by Lieb [18], Karamata's theorem yields

$$
\sum_{i=1}^{n} \varphi\left(\lambda_{i}(\varrho)\right) \leqq\left\langle\sum_{i=1}^{n} \varphi\left(a_{i}\right)\right\rangle_{\varrho}
$$

if $\varphi$ is convex non-decreasing, and

$$
\sum_{i: \lambda_{i} \geqq 0} \varphi\left(\lambda_{i}(\varrho)\right) \leqq\left\langle\sum_{i: a_{1} \geqq 0} \varphi\left(a_{i}\right)\right\rangle_{\varrho}
$$

if furthermore $\varphi(t)=0$ for $t \leqq 0$. The estimates (8), (9) yield rigorous bounds on the Hausdorff dimension and the entropy as follows from (5) and (6). 


\section{Application to Dissipative Physical Systems}

In order to define the characteristic exponents we want the operators $T(x, t)$ to be compact. In fact, in order to use the inequalities of Sect. 5, we want the eigenvalues $a_{i}$ of $\mathscr{H}(x)$ to satisfy $\lim _{i \rightarrow \infty} a_{i}=-\infty$. [These conditions can be relaxed a bit, see [31]. In particular, if the $T(x, t)$ are bounded but not compact one can still define the "largest characteristic exponent" by the theorem of Furstenberg and Kesten [10] which is a predecessor of the multiplicative ergodic theorem.]

In practice $\lim _{i \rightarrow \infty} a_{i}=-\infty$ in those cases where $\mathscr{H}$ contains a Laplace operator operating on a (vector-valued) function in a bounded domain. The Laplacian corresponds to a dissipative phenomenon: friction (diffusion of momentum), heat diffusion or ordinary diffusion. Unfortunately, compressible fluids are not allowed because the equation of conservation of mass:

$$
\frac{\partial \varrho}{\partial t}+\operatorname{div}(\varrho u)=0
$$

does not have a Laplacian acting on $\varrho$. The incompressibility condition $\operatorname{div} u=0$ is acceptable however as an auxiliary condition. Altogether, our inequalities will be useful for dissipative systems which are incompressible and enclosed in a bounded region ${ }^{11}$. Otherwise one may get a finite upper bound for the largest characteristic exponent, but divergent estimates for Hausdorff dimension and entropy.

\section{The Navier-Stokes Equation}

Our previous discussion applies to the characteristic exponents of a viscous incompressible fluid enclosed in a bounded region $\Omega$ in $d$ dimensions ( $d=2$ or 3 ). The Navier-Stokes equation is used:

$$
\frac{\partial u_{i}}{\partial t}=-\sum_{j} u_{j} \partial_{j} u_{i}+v \Delta u_{j}-\partial_{j} p+g_{i}, \sum_{i} \partial_{i} u_{i}=0 .
$$

(The velocity $u_{i}$ on the boundary $\partial \Omega$ is imposed). The operator $\mathscr{H}$ corresponding to the Navier-Stokes equation are of Schrödinger type. The distribution of the eigenvalues $a_{i}$ of $\mathscr{H}$ may be estimated by a classical approximation, which also yields rigorous inequalities, studied by Lieb and Thirring [19] (see also the review in Reed and Simon [28] IV Sect. XIII 15 and Fefferman [7]). The original study was made in Ruelle [32], and the results improved by Lieb [18]. One can further generalize by allowing time dependent forces. One obtains thus, for $\gamma \geqq 1$,

$$
\sum_{i: \lambda_{i} \geqq 0}\left(\lambda_{i}(\varrho)\right)^{\gamma} \leqq d\left(\frac{d-1}{2 d}\right)^{\gamma / 2+d / 4} L_{\gamma d} v^{-\gamma / 2-3 d / 4}\left\langle\int_{\Omega} \varepsilon_{u}^{\gamma / 2+d / 4}\right\rangle_{\varrho},
$$

where the $\varrho$-average is over the velocity field $u$, and

$$
\varepsilon_{u}=\frac{v}{2} \sum_{i, j}\left(\partial_{i} v_{j}+\partial_{j} v_{i}\right)^{2}
$$

11 I am indebted to U. Frisch, A. Lafon, and J. L. Lebowitz for discussions on these matters. It remains unclear to me if incompressible fluids are really excluded from the analysis, or if they could be handled by a suitable trick 
is the dissipation rate; $L_{\gamma d}$ is a finite constant. [The improvement by $\left(\frac{d-1}{d}\right)^{\gamma / 2+d / 4}$ over [32] is due to Lieb [18], who gives $L_{1,2} \leqq 0.24008$ and $L_{1,3} \leqq 0.040304$ as the current best estimates.

In particular, an estimate of the rate of information production is obtained by putting together the inequality (6) with (10) for $\gamma=1$ :

$$
h_{\mathbf{f}}(\varrho)-h_{\mathbf{g}}(\varrho) \leqq d\left(\frac{d-1}{2 d}\right)^{\gamma / 2+d / 4} L_{1 d} v^{-1 / 2-3 d / 4}\left\langle\int_{\Omega} \varepsilon_{u}^{1 / 2+d / 4}\right\rangle_{\varrho} .
$$

The function $c_{\varrho}$ defined in Sect. 4 satisfies (according to [18], Eqs. (1), (27))

$$
\begin{gathered}
c_{\varrho}(n d) \leqq d\left\langle\sup \left\{\tilde{F}(\varphi): \int \varphi=n, \varphi \geqq 0\right\}\right\rangle_{\varrho}, \\
\tilde{F}(\varphi)=-v K_{d} \int_{\Omega} \varphi^{p}+\int_{\Omega} \varphi \cdot\left(\frac{d-1}{2 v d} \varepsilon\right)^{1 / 2}, \\
p=1+2 / d,
\end{gathered}
$$

where $K_{d}$ is (for each $d$ ) a universal constant. The estimate (12) is obtained by comparing the eigenvalues of $\mathscr{H}$ with the eigenvalues of another operator repeated $d$ times. Therefore an upper bound to $c_{\varrho}(k)$ is obtained for any integer $k>0$ by linear interpolation of the right-hand side of $(12)$ between values $n d,(n+1) d$. Since the right-hand side is concave with respect to $n$ we have, for all real $s$,

$$
c_{\varrho}(s) \leqq d\left\langle\sup \left\{\widetilde{F}(\varphi): \int \varphi=\frac{s}{d}, \varphi \geqq 0\right\}\right\rangle_{\varrho} .
$$

Therefore the argument of Lieb (leading to [18], Eq. (43)) yields the following estimate for the Liapunov dimension:

$$
\frac{\operatorname{dim}_{\Lambda}(\varrho)}{|\Omega|} \leqq d\left(\frac{d-1}{2 d}\right)^{d / 4} A_{d} v^{-3 d / 4}\left(\left\langle\int \varepsilon^{1 / 2+d / 4} \frac{d x}{|\Omega|}\right\rangle_{\varrho}\right)^{\frac{d}{d+2}}
$$

[This result had been obtained in [32] only for $d=3$, and assuming the correctness of an unproved conjecture of Lieb and Thirring [19]. The best estimates on $A_{d}$ are currently [18]: $A_{2} \leqq 0.5597, A_{3} \leqq 0.1329$.]

The inequalities (10), (11), and (13) are quite general, and valid also when the boundaries $\partial \Omega$ of the region containing the fluid are time dependent. (This is the situation of a fluid agitated by a propeller; the case of a spoon in a cup of coffee is not allowed because of the coffee-air surface which makes this a more difficult problem.) The case of moving boundaries is not exactly of the form discussed earlier, because the region $\Omega$ and therefore the Hilbert space $M$ depend on time, but the extension is straightforward.

\section{The Navier-Stokes Equation in 2 Dimensions}

The situation of the existence and uniqueness problem for the Navier-Stokes equation is different in 2 and 3 dimensions. 
If $d=2$, there is a good existence and uniqueness theorem (Leray, Ladyzhenskaya). For time independent forces, a sufficiently large ball $U \subset M$ is mapped by time evolution to a compact set contained in $U$. There is therefore a universal attracting set $K$ such that every solution of the Navier-Stokes equation approaches $K$ when $t \rightarrow \infty$. (A nice discussion of this is given in Foias and Temam [8] Sect. 2, this article contains further references to Navier-Stokes theory ${ }^{12}$ ). For the more general case of time dependent forces there is a universal attracting family of compact sets $(K(y))_{y \in S}$.

We shall further discuss the case where the boundaries of $\Omega$ are fixed ( $u=0$ on $\partial \Omega)$. We have then the identity

$$
\frac{\partial}{\partial t}|u|^{2}=-2 v\|u\|^{2}+2(u, g),
$$

where || is the $L^{2}$ norm, and the Dirichlet norm \|\| is defined by $\|u\|^{2}=\sum_{i} \sum_{j} \int_{\Omega}\left(\partial_{i} u_{j}\right)^{2}$. Thus

$$
\left\langle\int_{\varepsilon}\right\rangle_{\varrho}=\left\langle v\|u\|^{2}\right\rangle_{\varrho} \leqq \frac{1}{v}\left\langle\|g\|^{\prime 2}\right\rangle_{\varrho},
$$

where \|\|$^{\prime}$ is the norm in the dual of the Dirichlet space with the norm \|\| . If $-\mu$ is the largest eigenvalue of the Laplacian in $\Omega$, we have ${ }^{13}$

$$
\|g\|^{2} \leqq \mu^{-1}|g|^{2}, \quad \mu \geqq \frac{2}{|\Omega|},
$$

where $|\Omega|$ is the area of $\Omega$, and therefore

$$
\left\langle\int_{\varepsilon} \varepsilon\right\rangle_{\varrho} \leqq \frac{|\Omega|}{2 v}\left\langle|g|^{2}\right\rangle_{\sigma} .
$$

Using (11), (13) we have in particular the entropy and Hausdorff dimension estimates $^{14}$

$$
\begin{gathered}
h_{\mathbf{f}}(\varrho)-h_{\mathbf{g}}(\sigma) \leqq \frac{1}{2} L_{12} v^{-2}\left\langle\int_{\Omega} \varepsilon\right\rangle_{\varrho} \leqq \frac{1}{4} L_{12} v^{-3}|\Omega|\left\langle|g|^{2}\right\rangle_{\sigma}=L_{12} \frac{v}{|\Omega|} \tilde{G}^{2} \\
\operatorname{dim}_{H} K(y) \leqq A_{2} v^{-3 / 2}|\Omega|^{1 / 2}\left(\left\langle\int_{\varepsilon} \varepsilon\right\rangle_{\varrho}\right)^{1 / 2} \leqq \frac{A_{2}}{\sqrt{2}} v^{-2}|\Omega|\left(\left\langle\left|g^{2}\right|\right\rangle_{\sigma}\right)^{1 / 2}=\sqrt{2} A_{2} \tilde{G} \\
L_{12} \leqq 0.24008, A_{2} \leqq 0.5597
\end{gathered}
$$

12 Standard references are Ladyzhenskaya [15], Lions [20], and Temam [36]; see also Tartar [35]

13 Note that $\iint u^{2} \leqq \int d x_{1} \max _{x_{2}}|u| \int d x_{2} \max _{x_{1}}|u| \leqq\left(\iint\left|\partial_{1} u\right|\right)\left(\iint\left|\partial_{2} u\right|\right)$

$$
\leqq\left(|\Omega| \iint\left|\partial_{1} u\right|^{2}\right)^{1 / 2}\left(|\Omega| \iint\left|\partial_{2} u\right|^{2}\right)^{1 / 2}=|\Omega|\left(\frac{1}{2} \iint\left(\partial_{i} u\right)\left(\partial_{i} u\right)\right)=\frac{1}{2}|\Omega|\|u\|^{2}
$$

14 In the case of time independent forces, $\sup h(\varrho)$ is the topological entropy, which is thus bounded above by $L_{12} \frac{v}{|\Omega|} \widetilde{G}^{2}$ 
where we have written $\widetilde{G}=\frac{|\Omega|}{2 v^{2}}\left(\left\langle|g|^{2}\right\rangle_{\sigma}\right)^{1 / 2}$ by analogy with the generalized Grasshof number $G=\frac{|g|}{v^{2} \mu}$ of Constantin and Foias [4]. We have $G \leqq \widetilde{G}$, and $G$ is proportional to $\widetilde{G}$ when $\Omega$ has a fixed shape. The estimate (15) improves the results of Constantin and Foias $\operatorname{dim}_{H} K \leqq \operatorname{const}\left((\log G)^{1 / 2}+1\right)$ in the case of periodic boundary conditions, and $\operatorname{dim}_{H} K \leqq$ const $G^{2}$ in general, with constants depending on the shape of $\Omega$ ).

An example of 2-dimensional fluid set into motion by boundary effects will be treated in Sect. 10 (convection).

\section{The Navier-Stokes Equation in 3 Dimensions}

If $d=3$ there is no longer an existence and uniqueness theorem. One has a unique solution for small times if the initial condition is good, and one can define "weak solutions" for all times, but without uniqueness (Leray [17], Hopf [13]). This is because the time evolution might create singularities even if the initial condition is smooth. One knows however that the singularities can occupy only a small subset of 4-dimensional space-time (see Leray [17], Scheffer [33], [34], and the best results in Caffarelli, Kohn, and Nirenberg [2]). It is in fact not known if singularities occur at all.

Here we shall ignore singularities, and assume that our initial conditions are in some open set $U$ which is mapped inside itself by time evolution. There is then an attracting family of compact sets $(K(y))_{y \in S}$ as in the 2-dimensional case. The entropy and Hausdorff dimension estimates are here

$$
\begin{gathered}
h_{\mathbf{f}}(\varrho)-h_{\mathbf{g}}(\sigma) \leqq \frac{1}{3^{1 / 4}} L_{13} v^{-11 / 4}\left\langle\int_{\Omega} \varepsilon^{5 / 4}\right\rangle_{\varrho}, \\
\operatorname{dim}_{H} K(y) \leqq 3^{1 / 4} A_{3} v^{-9 / 4}|\Omega|^{2 / 5}\left(\left\langle\int \varepsilon^{5 / 4}\right\rangle_{\varrho}\right)^{3 / 5}, \\
L_{13} \leqq 0.040304, A_{3} \leqq 0.1329,
\end{gathered}
$$

where $|\Omega|$ denotes the volume of $\Omega$.

It is not known how to bound $\left\langle\int \varepsilon^{5 / 4}\right\rangle_{e}$ in terms of the forces acting on the fluid (assuming $u=0$ on $\partial \Omega$ ). It is however possible to bound this quantity in terms of $\left\langle\left(\int \varepsilon\right)^{2}\right\rangle$ and the forces by using the inequalities ${ }^{15}$

$$
\begin{gathered}
\varepsilon^{3} \leqq \operatorname{const} v^{3}|\Delta u|^{6}, \\
\left\langle\left(\int \varepsilon\right)^{\alpha} v|\Delta u|^{2}\right\rangle_{\varrho} \leqq \operatorname{const} \frac{1}{v^{6}}\left\langle\left(\int \varepsilon\right)^{3+\alpha}\right\rangle_{\varrho}+v \frac{3+11 \alpha}{5}\left\langle\|g\| \frac{6+2 \alpha}{5}\right\rangle_{\sigma},
\end{gathered}
$$

15 Multiplying the Navier-Stokes equation by $\Delta u$, integrating, then multiplying by $\|u\|^{2 \alpha}$, one obtains

$$
\frac{1}{\alpha+1} \frac{d}{d t}\|u\|^{2+2 \alpha}+\|u\|^{2 \alpha} v|\Delta u|^{2 \alpha} \leqq \text { const } v^{-3}\|u\|^{6+2 \alpha}+2\|g\| \cdot\|u\|^{1+2 \alpha} .
$$

Averaging over time yields (18), which remains true for $\alpha=-1$ 
etc. Unfortunately these inequalities are not suited to a large volume limit because different terms are proportional to different powers of the volume of integration ${ }^{16}$.

\section{Convection}

We consider heat conduction by a viscous fluid, with gravity treated in the Boussinesq approximation. This means that the fluid is considered incompressible except for a buoyancy effect. We further assume that the various phenomenological and thermodynamic coefficients are constant ${ }^{17}$. Writing $\theta=T-T_{0}+\beta Z$, where $T_{0}$ is a reference temperature and $Z$ the vertical coordinate, we have the following evolution equations

$$
\begin{gathered}
\sum_{i} \partial_{i} u_{i}=0 \\
\frac{\partial u_{i}}{\partial t}=-\sum_{j} u_{j} \partial_{j} u_{i}+v \Delta u_{i}-\frac{1}{\varrho} \partial_{i} p+g \alpha \theta \partial_{i} Z \\
\frac{\partial \theta}{\partial t}=-\sum_{i} u_{i} \partial_{i}(\theta-\beta Z)+\kappa \Delta \theta+\frac{1}{c} \frac{v}{2} \sum_{i, j}\left(\partial_{i} u_{j}+\partial_{j} u_{i}\right)^{2}-\frac{1}{c} \sum_{i} u_{i} g \alpha \theta \partial_{i} Z .
\end{gathered}
$$

The term $g \alpha \theta \partial_{i} Z$ in (20) is the buoyant acceleration, defined only up to a gradient (absorbed in $\partial_{i} p$ ); $c$ is a specific heat. The fluid is enclosed in a box $\Omega$. The velocity and temperature are imposed on the boundary of $\Omega$, where it is assumed that $\left(u_{i}\right)$ and $\theta$ vanish. We simplify our problem by taking the specific heat $c$ infinite, so that (21) becomes

$$
\frac{\partial \theta}{\partial t}=-\sum_{i} u_{i} \partial_{i}(\theta-\beta Z)+\kappa \Delta \theta
$$

Furthermore we shall discuss only the 2-dimensional situation and take $\partial_{i} Z=\delta_{i 2}$. The linearized Eqs. (20), (21) are of the form

$$
\frac{\partial \xi}{\partial t}=\mathscr{K} \xi
$$

where $\xi$ is a vector field with components $U_{1}, U_{2}, k \Theta$, satisfying $\sum_{i} \partial_{i} U_{i}=0$. (The constant $k$ will be fixed later.) Define

$$
\|\xi\|^{2}=\int_{\Omega} \sum_{i} \xi_{i}^{2} .
$$

The self-adjoint part $\mathscr{H}$ of $\mathscr{K}$ is then defined by

$$
\begin{aligned}
(\xi, \mathscr{H} \xi)=(\xi, \mathscr{K} \xi)= & \int\left[v \sum_{i=1}^{2} U_{i} \Delta U_{i}-\sum_{i, j=1}^{2} U_{i} U_{j} \frac{1}{2}\left(\partial_{i} u_{j}+\partial_{j} u_{i}\right)+U_{2} g \alpha \Theta\right. \\
& \left.+\kappa k^{2} \Theta \Delta \Theta-k^{2} \Theta \sum_{i=1}^{2} U_{i} \partial_{i}(\theta-\beta Z)\right] \\
= & \int\left[v \sum_{i=1}^{2} \xi_{i} \Delta \xi_{i}+\kappa \xi_{3} \Delta \xi_{3}-\sum_{i, j=1}^{3} M_{i j} \xi_{i} \xi_{j}\right],
\end{aligned}
$$

16 R. Mañé has informed me that he has obtained Hausdorff dimension estimates (unpublished), Constantin, Foias, Manley, and Temam [5] have also announced such estimates

17 For a classical study of convection see Chandrasekhar [3]; see also Glansdorff and Prigogine [11], Chap. XI 
with

$$
\sum_{i, j=1}^{3} M_{i j} \xi_{i} \xi_{j}=\sum_{i, j=1}^{2} \frac{1}{2}\left(\partial_{i} u_{j}+\partial_{j} u_{i}\right) \xi_{i} \xi_{j}+k\left(\partial_{1} \theta\right) \xi_{1} \xi_{3}+\left(k \partial_{2} \theta-\frac{1}{k} g \alpha-k \beta\right) \xi_{2} \xi_{3} .
$$

The symmetric $3 \times 3$ matrix $M$ satisfies $\operatorname{Tr} M=0$, and therefore its eigenvalues are bounded by $\left(\frac{2}{3} \operatorname{Tr} M^{2}\right)^{1 / 2}$ (Lieb's remark, see [18]). Therefore

$$
\mathscr{H} \leqq \Delta \otimes\left(\begin{array}{lll}
v & & \\
& v & \\
& & \kappa
\end{array}\right)+\left(\frac{2}{3} \operatorname{Tr} M^{2}\right)^{1 / 2} \otimes\left(\begin{array}{lll}
1 & & \\
& 1 & \\
& & 1
\end{array}\right) .
$$

For a probability measure $\varrho$ ergodic under time evolution, we have then, by easy modification of standard arguments ([32] and [18]):

$$
\sum_{i: \lambda_{i} \geqq 0}\left(\lambda_{i}(\varrho)\right)^{\gamma} \leqq L_{\gamma 2}\left(2 v^{-1}+\kappa^{-1}\right)\left\langle\int\left(\frac{2}{3} \operatorname{Tr} M^{2}\right)^{1 / 2(\gamma+1)}\right\rangle_{\varrho}
$$

for $\gamma \geqq 1$, and

$$
\operatorname{dim}_{\Lambda}(\varrho) \leqq A_{2}\left(2 v^{-1}+\kappa^{-1}\right)|\Omega|^{1 / 2}\left\langle\int \frac{2}{3} \operatorname{Tr} M^{2}\right\rangle_{\varrho}^{1 / 2}
$$

We have

$$
\int \operatorname{Tr} M^{2}=\int\left[\frac{1}{2} \sum_{i, j=1}^{2}\left(\partial_{i} u_{j}\right)^{2}+\frac{k^{2}}{4} \sum_{i=1}^{2}\left(\partial_{i} \theta\right)^{2}+\frac{1}{4}\left(\frac{g \alpha}{k}+k \beta\right)^{2}\right],
$$

because cross products disappear by integration. On the other hand (20) and (22) yield

$$
\begin{gathered}
v\left\langle\int \sum_{i, j=1}^{2}\left(\partial_{i} u_{j}\right)^{2}\right\rangle_{\varrho}=g \alpha\left\langle\int u_{2} \theta\right\rangle_{\varrho}, \\
\kappa\left\langle\int \sum_{i=1}^{2}\left(\partial_{i} \theta\right)^{2}\right\rangle_{\varrho}=\beta\left\langle\int u_{2} \theta\right\rangle_{\varrho},
\end{gathered}
$$

so that

$$
\left\langle\int \operatorname{Tr} M^{2}\right\rangle=\int\left[\left(\frac{g \alpha}{2 v}+\frac{k^{2} \beta}{4 \kappa}\right)\left\langle\int u_{2} \theta\right\rangle+\frac{1}{4}\left(\frac{g \alpha}{k}+k \beta\right)^{2}\right],
$$

and taking $k=(g \alpha / \beta)^{1 / 2}$, we get

$$
\left\langle\int \operatorname{Tr} M^{2}\right\rangle=g \alpha\left[\frac{1}{4}\left(2 v^{-1}+\kappa^{-1}\right)\left\langle\int u_{2} \theta\right\rangle+\beta|\Omega|\right] .
$$

We specialize now to the case of a rectangular region $\Omega$ of height $h$ and basis $b$, so that $|\Omega|=b h$, and $Z$ varies from 0 to $h$. We assume that the initial temperature $T$ in $\Omega$ is between $T_{0}$ and $T_{0}-\beta h$; the evolution equations imply that this remains true at all times, and we have

$$
-\beta(h-Z) \leqq \theta \leqq \beta Z .
$$

Writing $\theta_{ \pm}=\frac{1}{2}(|\theta| \pm \theta)$, we have

$$
\int_{0}^{h} u_{2} \theta d Z=\int_{0}^{h} u_{2} \frac{d}{d Z}\left(\int_{0}^{Z} \theta_{+}+\int_{Z}^{h} \theta_{-}\right),
$$


where

$$
0 \leqq \int_{0}^{Z} \theta_{+}+\int_{Z}^{h} \theta_{-} \leqq \frac{1}{2} \beta\left(Z^{2}+(Z-h)^{2}\right) \leqq \frac{1}{2} \beta h^{2} \leqq 2 \cdot \frac{1}{2} \beta\left(Z^{2}+(Z-h)^{2}\right) .
$$

Therefore

$$
\int_{0}^{h} u_{2} \theta d Z \leqq \int_{0}^{h}\left(\partial_{2} u_{2}\right)_{+} \cdot \frac{1}{2} \beta\left(Z^{2}+(Z-h)^{2}\right) d Z \leqq \frac{2}{3} \int_{0}^{h}\left|\partial_{2} u_{2}\right| \frac{1}{2} \beta\left(Z^{2}+(Z-h)^{2}\right) d Z
$$

(where we have used $\left.\int_{0}^{h} \partial_{2} u_{2} d Z=0\right)$, hence

$\int_{0}^{h} u_{2} \theta d Z \leqq \frac{1}{3}\left(\frac{7}{15}\right)^{1 / 2} \beta h^{5 / 2}\left(\int_{0}^{h}\left(\partial_{2} u_{2}\right)^{2} d Z\right)^{1 / 2} \leqq \frac{1}{2}\left[\frac{7}{270 v} g \alpha \beta^{2} h^{5}+\frac{2 v}{g \alpha} \int_{0}^{h}\left(\partial_{2} u_{2}\right)^{2} d Z\right]$,

hence

$$
g \alpha \int_{\Omega} u_{2} \theta \leqq \frac{1}{2} \cdot \frac{7}{270 v} g^{2} \alpha^{2} \beta^{2} h^{4}|\Omega|+\frac{v}{2} \int_{\Omega} \sum_{i, j=1}^{2}\left(\partial_{i} u_{j}\right)^{2},
$$

where we have used the fact that $\left(\partial_{1} u_{1}\right)^{2}=\left(\partial_{2} u_{2}\right)^{2}$ as a consequence of (19). In view of (25) we have thus

$$
g \alpha\left\langle\int u_{2} \theta\right\rangle \leqq \frac{7}{270 v} g^{2} \alpha^{2} \beta^{2} h^{4}|\Omega|
$$

Therefore (26) yields

$$
\left\langle\int \frac{2}{3} \operatorname{Tr} M^{2}\right\rangle \leqq\left[\frac{7}{1620}(1+2 \kappa / v) \frac{g \alpha \beta h^{4}}{\kappa v}+\frac{2}{3}\right] g \alpha \beta|\Omega|
$$

and we obtain

$$
\begin{gathered}
\sup _{\varrho} h(\varrho) \leqq \sup _{\varrho} \sum_{i: \lambda_{i} \geqq 0} \lambda_{i}(\varrho) \leqq L_{12}\left[\frac{7}{1620}(1+2 \kappa / v) \frac{g \alpha \beta h^{4}}{\kappa v}+\frac{2}{3}\right]\left(2 v^{-1}+\kappa^{-1}\right) g \alpha \beta|\Omega| \\
=L_{12}\left[\frac{7}{1620} \mathscr{R}+\frac{2}{3}\right] \mathscr{R} \cdot(v+2 \kappa) \frac{|\Omega|}{h^{4}} \\
\sup _{\varrho} \operatorname{dim}_{\Lambda}(\varrho) \leqq A_{2}\left[\frac{7}{1620}(1+2 \kappa / v) \frac{g \alpha \beta h^{4}}{\kappa v}+\frac{2}{3}\right]^{1 / 2}\left(2 v^{-1}+\kappa^{-1}\right)|\Omega|(g \alpha \beta)^{1 / 2} \\
=A_{2}\left[\frac{7}{1620}(1+2 \kappa / v) \mathscr{R}+\frac{2}{3}\right]^{1 / 2} \mathscr{R}^{1 / 2}\left(2\left(\frac{\kappa}{v}\right)^{1 / 2}+\left(\frac{v}{\kappa}\right)^{1 / 2}\right) \frac{|\Omega|}{h^{2}}
\end{gathered}
$$

where we have introduced the Rayleigh number $\mathscr{R}=\frac{g \alpha \beta}{\kappa v} h^{4}$.

If $K$ is the universal attracting set for the present problem ${ }^{18}, \operatorname{dim}_{H} K$ is bounded by (28) while the topological entropy of the time evolution (restricted to $K$ ), i.e., $\operatorname{suph}(\varrho)$, is bounded by (27). In particular, we have an upper bound to the

18 The existence of such a set is fairly clear, but we have in fact not proved it 
dimension of $K$ which is linear in $\mathscr{R}$. This means physically that the number of "excited modes" does not grow faster than linearly with the imposed temperature gradient.

\section{Conclusion}

In this paper, we have described a relatively systematic method of obtaining upper bounds on characteristic exponents, entropy and Hausdorff dimension for a time evolution described by nonlinear partial differential equations. The full nonlinear problem is decomposed into a linear part and a nonlinear part. The linear problem can be handled systematically by use of existing methods (of the type of classical estimates for a quantum system). The remaining nonlinear problem requires making suitable a priori estimates. The method has been illustrated by examples from hydrodynamics, but it is potentially useful in the study of many dissipative physical systems.

\section{Appendix}

In this appendix we discuss a situation slightly more general than in the main text: we allow $M$ to be a manifold. On the other hand the time is taken to be discrete, which is sufficient (use a time one map).

Let $M$ be a Hilbert manifold (modelled on a separable Hilbert space) and $S$ a compact metrizable space. Let also $\varrho$ be a Radon probability measure on the compact set supp $\varrho$ (the support of $\varrho$ ) contained in $M \times S$. We denote by $\pi$ : supp $\varrho \rightarrow S$ the canonical projection. We assume that there is a neighborhood $U \times S$ of supp $\varrho$ in $M \times S$, and continuous maps f: $U \times S \rightarrow M \times S, \mathbf{g}: S \rightarrow S$, such that f supp $\varrho=\operatorname{supp} \varrho, \mathbf{f} \varrho=\varrho$, and $\pi \circ \mathbf{f}=\mathbf{g} \circ \pi$. We write $\mathbf{f}(x, y)=\left(f_{y} x, \mathbf{g} y\right)$, and $\sigma=\pi \varrho$.

\section{Disintegration}

In the above setup we can disintegrate $\varrho$ with respect to $\pi$, i.e. $\int \varrho(d x d y)=\int \sigma(d y) \varrho_{y}(d x)$, where $\varrho_{y}$ is a probability measure with support in $U$ defined for $\sigma$-almost all $y$. [See for instance Bourbaki [1], Integration Chap. 6 Sect. 3 No. 1.] We have the invariance relation $f_{y} \varrho_{y}=\varrho_{g y}$.

Assume now that the maps $f_{y}: U \rightarrow M$ are of class $C^{1}$ and define the linear map $T_{(x, y)}$ to be the tangent of $f_{y}$ at the point $x$. Therefore $T_{(x, y)}$ maps the tangent space $T_{x} M$ to $T_{x^{\prime}}, M$, where $x^{\prime}=f_{y} x$, and $T_{(x, y)}$ depends continuously on $x$. We assume that $T_{(x, y)}$ depends continuously on $(x, y) \in U \times S$. Using a finite number of charts of $M$, one may consider all $T_{(x, y)}$ as acting on a single Hilbert space, but the dependence on $(x, y)$ will in general only be measurable, instead of continuous.

\section{Multiplicative Ergodic Theorem ${ }^{19}$}

From now on we assume that the operators $T_{(x, y)}$ are compact, and write

$$
T_{(x, y)}^{n}=T_{\mathbf{f}^{n-1}(x, y)} \ldots T_{\mathbf{f}(x, y)} T_{(x, y)} .
$$

19 For this infinite dimensional variant of the multiplicative ergodic theorem of Oseledec, see Ruelle [31], Mañé [24] 
Then, for $\varrho$-almost all $(x, y)$, the following limit exists in norm and is compact:

$$
\lim _{n \rightarrow \infty}\left(T_{(x, y)}^{n} * T_{(x, y)}^{n}\right)^{1 / 2 n}=\Lambda_{(x, y)}
$$

(here $T^{*}$ is the adjoint of $T$ ). Let $\exp \lambda^{(1)}>\ldots>\exp \lambda^{(j)}>\ldots$ be the sequence of eigenvalues of $\Lambda_{(x, y)}$, and $U_{(x, y)}^{(1)}, \ldots, U_{(x, y)}^{(j)}, \ldots$ the corresponding eigenspaces. If $V_{(x, y)}^{(r)}$ is the orthogonal complement of $U^{(1)}+\ldots+U^{(r-1)}$, then

$$
\lim _{n \rightarrow \infty} \frac{1}{n} \log \left\|T_{(x, y)}^{n} u\right\|=\lambda_{(x, y)}^{(r)}, \quad \text { when } \quad u \in V^{(r)} \backslash V^{(r+1)}
$$

for $r=1, \ldots$ (and the $(x, y)$ for which $(*)$ holds).

The $\lambda^{(j)}$ are the characteristic exponents, and their multiplicities are the $m^{(j)}=\operatorname{dim} U^{(j)}$. If $\varrho$ is $\mathbf{f}$-ergodic the characteristic exponents and their multiplicities are $\varrho$-almost everywhere constant. Let $\lambda_{1}(\varrho) \geqq \lambda_{2}(\varrho) \geqq \ldots$ be these characteristic exponents repeated according to multiplicity, define

$$
c_{\varrho}(n)=\sum_{i}^{n} \lambda_{i}(\varrho) \quad \text { for integer } n \geqq 0,
$$

and extend $c_{\varrho}$ to be linear on intervals $[n, n+1]$. Notice that $c_{\varrho}$ is concave on $[0,+\infty)$, that $c_{\varrho}(0)=0$, and that $c_{\varrho}(s)$ may take the value $-\infty$ for suffiently large $s$. Define also

$$
\operatorname{dim}_{\Lambda}(\varrho)=\max \left\{s: c_{\varrho}(s) \geqq 0\right\}
$$

\section{Hausdorff Dimension}

Remember that $\mathbf{f}(x, y), T_{(x, y)}$ depend continuously on $(x, y) \in U \times S$. We assume that there is a sequence $\left(y_{(-n)}\right)_{n \geqq 0}$ such that $\mathbf{g}^{m} y_{(-n)}=y_{(m-n)}$, and

$$
\underset{n \rightarrow \infty}{\operatorname{vague}} \lim \frac{1}{n} \sum_{0}^{n-1} \delta_{(-i)}=\sigma \text {. }
$$

We also assume that there is a compact $K \subset U$ such that $f_{y(-i)} K \subset K$, and define

Then

$$
K(y(0))=\bigcap_{i \geqq 0} f_{y(-i)} K .
$$

$\operatorname{dim}_{H} K(y(0)) \leqq \sup \left\{\operatorname{dim}_{\Lambda}(\varrho): \varrho\right.$ is ergodic, supp $\varrho \subset K \times S$ and $\left.\pi \varrho=\sigma\right\}$.

[When $S$ consists of a single point, this is a corollary (see Ledrappier [16], Corollary of Proposition 2) of a theorem of Douady and Oesterlé [6]. (Ledrappier's result is finite dimensional but, as he points out, it extends to infinite dimension. Related results have been obtained by Mañé.) The arguments of Douady, Oesterlé and Ledrappier extend in a straightforward manner to the present situation.]

\section{Entropy}

Assume again that $\varrho$ is ergodic. Then

$$
h_{\mathbf{f}}(\varrho)-h_{\mathbf{g}}(\sigma) \leqq \sum_{i: \lambda_{i} \geqq 0} \lambda_{i}(\varrho) .
$$


[This has been proved in [29] for the case when $M$ is finite dimensional and $S$ is a point. If $(S, \sigma)$ is non-trivial, replacing the entropy by the relative entropy is done in standard ways (see Jacobs [14], Sect. 10.5, Theorem 5). If $M$ has infinite dimension, the proof of finite Hausdorff dimension of $K(y)$ supplies coverings of $K(y)$ by very flat ellipsoids, which can be cut in small rectangular cells, and the argument of [29] extends to this situation. Another proof has been promised by R. Mañé.]

Acknowledgements. I am indebted to U. Frisch, A. Lafon, J.L. Lebowitz, R. Temam, and especially J.-P. Eckmann, F. Ledrappier, E. Lieb, and R. Mañé for discussions pertaining to the contents of the present paper.

\section{References}

1. Bourbaki, N.: Elements de Mathématiques, Livre VI. Intégration. Chap. 6. Intégration vectorielle, A.S.I. 1281, Paris: Herman 1959

2. Caffarelli, L., Kohn, R., Nirenberg, L.: Partial regularity of suitable weak solutions of the Navier-Stokes equations. Commun. Pure Appl. Math. 35, 771-831 (1982)

3. Chandrasekhar, S.: Hydrodynamic and hydromagnetic stability. Oxford: Clarendon 1961

4. Constantin, P., Foias, C.: Global Lyapunov exponents, Kaplan-Yorke formulas and the dimension of the attractors for $2 D$ Navier-Stokes equations. (to appear)

5. Constantin, P., Foias, C., Manley, O., Temam, R.: Analyse mathématique et mécanique des fluides - connection entre la théorie mathématique des équations de Navier-Stokes et la théorie conventionnelle de la turbulence. CRAS Paris (to appear)

6. Douady, A., Oesterlé, J.: Dimension de Hausdorff des attracteurs. C.R. Acad. Sci. Paris 290 A, 1135-1138 (1980)

7. Fefferman, C.: The uncertainty principle. Bull. Am. Math. Soc. 9, 129-206 (1983)

8. Foias, C., Temam, R.: Some analytic and geometric properties of the solutions of the evolution Navier-Stokes equations. J. Math. Pures Appl. 58, 339-368 (1979)

9. Frederikson, P., Kaplan, J.L., Yorke, J.A.: The dimension of the strange attractor for a class of difference systems. Preprint

10. Furstenberg, H., Kesten, H.: Products of random matrices. Ann. Math. Stat. 31, 457-469 (1960)

11. Glansdorff, P., Prigogine, I.: Structure, stabilité et fluctuations. Paris: Masson 1971

12. Grassberger, P., Procaccia, I.: Characterization of strange attractors. Phys. Rev. Lett. 50, 346-349 (1983)

13. Hopf, E.: Über die Anfangswertaufgabe für die hydrodynamischen Grundgleichungen. Math. Nachrichten 4, 213-331 (1951)

14. Jacobs, K.: Lecture notes on ergodic theory. Aarhus Universitet, Aarhus, 1963

15. Ladyzhenskaya, O.A.: The mathematical theory of viscous incompressible flow (2nd edn.), Moscow: Nauka, 1970, 2nd English edn. New York: Gordon and Breach 1969

16. Ledrappier, F.: Some relations between dimension and Lyapounov exponents. Commun. Math. Phys. 81, 229-238 (1981)

17. Leray, J.: Sur le mouvement d'un liquide visqueux emplissant l'espace. Acta Math.63, 193-248 (1934)

18. Lieb, E.: On characteristic exponents in turbulence. Commun. Math. Phys. 92, 473-480 (1984)

19. Lieb, E., Thirring, W.: Inequalitics for the moments of the eigenvalues of the Schrödinger equation and their relation to Sobolev inequalities, pp. 269-303. In: Studies in Mathematical Physics: Essays in Honor of Valentine Bargman. Licb, E., Simon, B., Wightman, A.S. (eds.), Princeton, N.J.: Princeton University Press 1976

20. Lions, J.L.: Quelques méthodes de résolution des problèmes aux limites non-linéaires, Paris: Dunod 1969

21. Mallet-Paret, J.: Negatively invariant sets of compact maps and an extension of a theorem of Cartwright. J. Diff. Eq. 22, 331-348 (1976) 
22. Malraison, B., Atten, P., Bergé, P., Dubois, M.: Dimension of strange attractors: an experimental determination for the chaotic regime of two convective systems. J. Phys. Lett. 44, L-897-902 (1983)

23. Mañé, R.: On the dimension of the compact invariant sets of certain nonlinear maps, pp. 230-242. In: Dynamical systems and turbulence, Warwick 1980, Lecture Notes in Mathematics, Vol. 898, New York: Springer 1981

24. Mañé, R.: Lyapounov exponents and stable manifolds for compact transformations, pp. 522-577. In: Geometric dynamics. Lecture Notes in Mathematics, Vol. 1007. Berlin, Heidelberg, New York: Springer 1983

25. McCluskey, H., Manning, A.: Hausdorff dimension for horseshoes, Ergod. Th. and Dynam. Syst. 3, 251-260 (1983)

26. Oseledec, V.I.: A multiplicative ergodic theorem. Lyapunov characteristic numbers for dynamical systems. Tr. Mosk. Mat. Obšč. 19, 179-210 (1968), English translation, Trans. Moscow Math. Soc. 19, 197-221 (1968)

27. Raghunathan, M.S.: A proof of Oseledec' multiplicative ergodic theorem. Israel J. Math. 32, 356-362 (1979)

28. Reed, M., Simon, B.: Methods of modern mathematical physics. New York: Academic Press 1972 (I), 1975 (II), 1979 (III), 1978 (IV)

29. Ruelle, D.: An inequality for the entropy of differentiable maps. Bol. Soc. Bras. Mat. 9, 83-87 (1978)

30. Ruelle, D.: Ergodic theory of differentiable dynamical systems. Publ. Math. IHES 50, 275-306 (1979)

31. Ruelle, D.: Characteristic exponents and invariant manifolds in Hilbert space. Ann. Math. 115, 243-290 (1982)

32. Ruelle, D.: Large volume limit of the distribution of characteristic exponents in turbulence. Commun. Math. Phys. 87, 287-302 (1982)

33. Scheffer, V.: Turbulence and Hausdorff dimension, pp. 174-183. In: Turbulence and NavierStokes equation, Lecture Notes in Mathematics, Vol. 565, Berlin: Springer 1976

34. Scheffer, V.: The Navier-Stokes equations on a bounded domain. Commun. Math. Phys. 73, $1-42(1980)$

35. Tartar, L.: Topics in nonlinear analysis. Publications mathématiques d'Orsay 78.13, Orsay, 1978

36. Temam, R.: Navier-Stokes equations. Revised edition, Amsterdam: North-Holland 1979

37. Young, L.-S.: Dimension, entropy and Lyapounov exponents. Ergod. Theor. Dynam. Syst. 2 , 109-124 (1982)

Communicated by A. Jaffe

Received January 13, 1984

Note added in proof: The following recent references are relevant to the subject matter of this paper:

[A] Babin, A.V.,Vishik, M.I.: Attractors for partial differential equations of evolution and estimation of their dimension. Usp. Mat. Nauk 38 No. 4 (232), 133-182 (1983)

[B] Foias, C., Manley, O.P., Temam, R., Trève, Y.M.: Asymptotic analysis of the NavierStokes equation. Physica 9 D, 157-188 (1983)

In particular $[\mathrm{A}]$ contains lower bounds on the dimension of attracting sets (obtained by proving the existence of an unstable fixed point). 